\title{
Expanding the investigation of meaningful effects in physiology research
}

\author{
"Magnitude-based inferences and the accurate \\ quantification of individual response differences \\ represent two recent statistical developments for the \\ evaluation of physiological outcomes."
}

First draft submitted: 5 May 2017; Accepted for publication: 10 May 2017; Published online: 7 July 2017

Keywords: appetite $\bullet$ confidence intervals $\bullet$ individual differences $\bullet$ magnitude-based inferences $\bullet$ nonresponders $\bullet$ personalized medicine $\bullet$ physiology $\bullet$ responders $\bullet$ significance testing $\bullet$ statistics

The statistical investigation of meaningful changes in response to physiological interventions has increased considerably during the past decade. Indeed, in the field of exercise physiology it is now commonplace for performance test outcomes to be assessed using magnitude-based inferences (MBI) as either the sole method of statistical analysis [1] or in combination with null-hypothesis significance testing [2]. Additionally, the focus on 'personalized medicine' during recent years has stimulated significant interest in the quantification of true and meaningful individual responses to interventions within the field of human physiology. The purpose of the present article is to provide a brief overview of MBI and individual response differences, with a focus on the potential for wider applications in other areas of physiology research. Recent developments from our research groups are used as examples to demonstrate the potential for an expanded use of these approaches.

\section{Investigating meaningful effects at the group level}

The MBI method derives the probability that an effect is beneficial, harmful or trivial based on the observed effect and its uncertainty in relation to a predetermined value representing a minimum clinically or practically important value of the effect [3]. This differs from null-hypothesis significance testing which assesses the span of confidence intervals (CIs) in relation to a 'null' effect (i.e., if the CIs of the effect do not span zero then the effect is deemed 'significant'). Rather than assessing significant differences, MBI provides an interpretation of the magnitude of changes and whether these are meaningful, which represents an intuitive approach for many researchers [4]. Assessing the magnitude of change in a probabilistic manner also reduces inferential error rates, increases the proportion of decisive (publishable) outcomes, and reduces publication bias, especially with small sample sizes [3].

The implementation of MBI for analysis of an intervention requires determination of a value for the smallest meaningful change in the relevant variable. To achieve this, it is often preferable to use a pre-established value informed by the literature which represents a practical or clinical benefit. Such values have been established for a range of variables in relation to minimum clinically important differences (e.g., the 6-min walk test in patients with chronic obstructive pulmonary disease [5]) or practical benefits (e.g., changes in athletic performance tests [6]). The recent incorporation of MBI to investigate changes in appetite perceptions in response to an acute exercise and nutritional intervention [7] utilized a well-established threshold for practically relevant changes of $8-10 \mathrm{~mm}$ when assessed using a $100-\mathrm{mm}$ visual analogue
Kevin Deighton

Author for correspondence: Institute for Sport, Physical Activity \& Leisure, Leeds Beckett University, Leeds, LS6 3QS, UK

Tel.: +440 01138123582

K.Deighton@leedsbeckett.ac.uk

\section{James A King}

School of Sport, Exercise \& Health Sciences, Loughborough University, Loughborough, LE11 3TU, UK

\section{David J Stensel}

School of Sport, Exercise \& Health Sciences, Loughborough University, Loughborough, LE11 3TU, UK

\section{Ben Jones}

Institute for Sport, Physical Activity \& Leisure, Leeds Beckett University, Leeds, LS6 3QS, UK 
scale [8]. This represents the first use of MBI in the analysis of appetite perceptions and highlights the potential wider utility of this approach in physiology research.

"The novel focus on these aspects of analysis in appetite research demonstrates the potential for more widespread use to assess a range of variables across a variety of research topics.

In addition to the approach described above, fractions of the between-subject SD may also be used as the value for the smallest meaningful change in the relevant variable (e.g., $20 \%$ of the between-subject SD would represent the threshold for a small effect size of 0.2 based on Cohen's $d$ ) [6]. This method represents a reasonable starting point for the assessment of novel variables in the absence of established meaningful change values of practical or clinical relevance.

\section{Investigating meaningful individual responses}

In combination with the assessment of effects at the group level, investigations into individual response differences have become prevalent within physiology research. This approach typically classifies participants as either 'responders' or 'nonresponders' based on the direction or magnitude of their individual response to an intervention [9,10]. Further statistical analyses or additional research studies are then sometimes performed to elucidate the reasons for these divergent responses. For example, this may involve an investigation into the participant characteristics of 'responders' compared with 'nonresponders', or further investigations into the underlying physiology of these groups of participants. However, this approach to classifying individual response differences does not account for random within-subject variation, which is comprised of natural biological variation between measurement points and the technical error from the measurement tool/protocol [9,11,12]. In a recent publication, Atkinson \& Batterham [9] provided a comprehensive overview of the potential influence of random within-subject variation on the measurement of physiological variables and demonstrated that this variation can sometimes account entirely for the apparent individual response differences observed. To remove the influence of random within-subject variation, true individual response differences require the $\mathrm{SD}$ of changes in response to an intervention to be greater than the same SD in a comparator arm (for randomized controlled trials) or from a prior reliability study (for crossover trials) [9]. The magnitude of this difference must be either practically or clinically relevant before mediators of this effect are to be examined [9].
The work of Atkinson \& Batterham [9] has emphasized the need for researchers to understand the random within-subject variation for a range of physiological measures before attempting to investigate individual response differences. Considering that random within-subject biological variation is likely to increase as the time period between trials becomes longer [9,13], it is important that acute crossover studies utilize reliability data from investigations that have separated trials by a similar period of time. The recruitment of similar participant populations is also important to increase the relevance and accuracy of reliability data. Accordingly, reliability studies have recently been employed within appetite research to determine individual differences in the appetite and energy intake responses to exercise [14] and food consumption [15]. Additionally, the work by King et al. [14] determined the within-subject variation in plasma acylated ghrelin concentrations as a mechanistic variable for understanding changes in appetite perceptions. This focus to understand meaningful individual responses in mechanistic and primary outcome measures may represent a useful model for other areas of physiology research. These studies also highlight the topical nature of investigations to understand random within-subject variation to provide a platform for the accurate assessment of true and meaningful individual response differences. Further investigation of other physiological variables is required, in addition to the examination of whether individual responses remain stable with repeated exposures to an intervention $[15,16]$.

\section{Conclusion \& future perspective}

MBI and the accurate quantification of individual response differences represent two recent statistical developments for the evaluation of physiological outcomes. The novel focus on these aspects of analysis in appetite research demonstrates the potential for more widespread use to assess a range of variables across a variety of research topics. Indeed, the integration of MBI within statistical analysis can be readily achieved by the determination of smallest meaningful change values as either a fraction of the between-subject SD or using established thresholds of practical or clinical relevance. Equally, with the increased focus on personalized medicine and nutrition, it is important for researchers to accurately assess true and meaningful individual response differences before conducting further research or providing a personalized intervention. We anticipate that the prevalence of these statistical approaches will increase in the coming years across a wider range of research topics. 


\section{Financial \& competing interests disclosure}

The authors have no relevant affiliations or financial involvement with any organization or entity with a financial interest in or financial conflict with the subject matter or materials discussed in the manuscript. This includes employment, consultancies, honoraria, stock ownership or options, expert testimony, grants or patents received or pending, or royalties.

\section{References}

1 Rowlands DS, Swift M, Ros M, Green JG. Composite versus single transportable carbohydrate solution enhances race and laboratory cycling performance. Appl. Physiol. Nutr. Metab. 37, 425-436 (2012).

2 de Salles Painelli V, Roschel H, Jesus FD et al. The ergogenic effect of beta-alanine combined with sodium bicarbonate on high-intensity swimming performance. Appl. Physiol. Nutr. Metab. 38, 525-532 (2013).

3 Hopkins WG, Batterham AM. Error rates, decisive outcomes and publication bias with several inferential methods. Sports Med. 46, 1563-1573 (2016).

4 Buchheit $\mathrm{M}$. The numbers will love you back in return-I promise. Int. J. Sports Physiol. Perform. 11, 551-554 (2016).

5 Polkey MI, Spruit MA, Edwards LD et al. Six-minute-walk test in chronic obstructive pulmonary disease: minimal clinically important difference for death or hospitalization. Am. J. Respir. Crit. Care Med. 187, 382-386 (2013).

6 Hopkins WG. How to interpret changes in an athletic performance test. Sports Sci. 8, 1-7 (2004).

7 Deighton K, Duckworth L, Matu J et al. Mouth rinsing with a sweet solution increases energy expenditure and decreases appetite during $60 \mathrm{~min}$ of self-regulated walking exercise. Appl. Physiol. Nutr. Metab. 41, 1255-1261 (2016).

8 Blundell J, de Graaf C, Hulshof T et al. Appetite control: methodological aspects of the evaluation of foods. Obes. Rev. 11, 251-270 (2010).
No writing assistance was utilized in the production of this manuscript.

\section{Open access}

This work is licensed under the Creative Commons Attribution 4.0 License. To view a copy of this license, visit http://creativecommons.org/licenses/by/4.0/

9 Atkinson G, Batterham AM. True and false interindividual differences in the physiological response to an intervention. Exp. Physiol. 100, 577-588 (2015).

10 Betts JA, Gonzalez JT. Personalised nutrition: what makes you so special? Nutr. Bull. 41, 353-359 (2016).

11 Hopkins WG, Marshall SW, Batterham AM, Hanin J. Progressive statistics for studies in sports medicine and exercise science. Med. Sci. Sports Exerc. 41, 3-12 (2009).

12 Hopkins WG. Individual responses made easy. J. Appl. Physiol. 118, 1444-1446 (2015).

13 Hopkins WG. Measures of reliability in sports medicine and science. Sports Med. 30, 1-15 (2000).

14 King JA, Deighton K, Broom DR et al. Individual variation in hunger, energy intake and ghrelin responses to acute exercise. Med. Sci. Sports Exerc. doi:10.1249/MSS.0000000000001220 (2017) (Epub ahead of print).

15 Gonzalez JT, Frampton J, Deighton K. Postprandial suppression of appetite is more reproducible at a group than an individual-level: implications for assessing inter-individual variability. Appetite 108, 375-382 (2017).

16 Hecksteden A, Kraushaar J, Scharhag-Rosenberger F, Thiesen D, Senn S, Meyer T. Individual responses to exercise training - a statistical perspective. J. Appl. Physiol. 118, 1450-1459 (2015). 